\section{Nie mehr eine Applikation vergessen}

D er Erfolg einer spezifischen Immuntherapie hängt ganz entscheidend von einer fortlaufenden Behandlung über mindestens drei Jahre $a b$. Um die Arzt-Patienten-Beziehung zu unterstützen, den Arzt zu entlasten und die Therapietreue des Patienten weiter

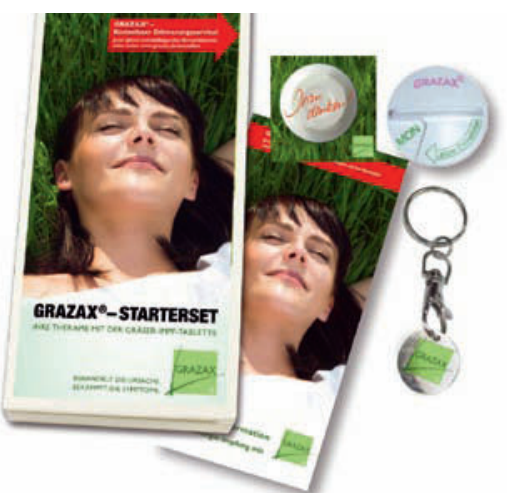

Das neue ALK-Compliance-Set gibt es in zwei Ausführungen: einmal für Erwachsene ... zu verbessern, hat das Pharmaunternehmen ALK nun den „ALK-ComplianceService" entwickelt. Ziel ist es, beim Patient ein besseres Verständnis der Therapie zu erreichen, deren positiven Einfluss auf die Symptome und damit die Lebensqualität zu vermitteln sowie über die Prävention eines Etagenwechsels aufzuklären.

Der ALK-Compliance-Service deckt alle Formen der Hyposensibilisierung ab. Passend zur jeweiligen Therapie und abhängig vom Alter des Patienten bestehen die Compliance-Pakete aus Patientenbroschüren, die den Therapieverlauf allgemeinverständlich beschreiben und erklären, sowie aus Erinnerungshilfen. Ein spezieller Service fordert außerdem per E-Mail oder SMS zur Arzneimitteleinnahme auf.

Der Vorteil für den Arzt besteht im einfachen Handling: Die Arzthelferin füllt nach der Erstverordnung einen

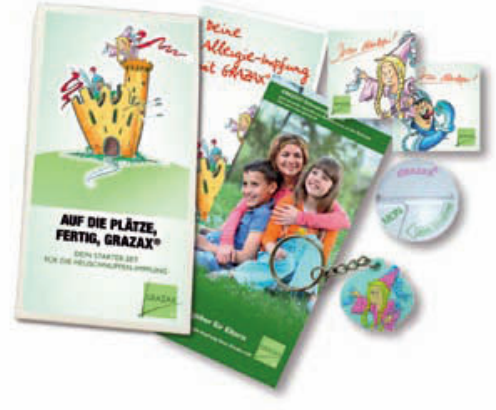

... und einmal für Kinder.

kurzen Bestellbogen aus. Das Compliance-Paket erhält der Patient dann per Post. Je nachdem, ob er sich für den SMS- oder E-Mail-Service entscheidet, wird er regelmäßig zur gewünschten Zeit an die Einnahme der Tabletten oder Tropfen erinnert. Auf Wunsch kann sich der Patient auch an die Folgeverordnung der subkutanen Immuntherapie erinnern lassen.

red

Nach Informationen von ALK-Abelló, Wedel

\title{
Ankunft in der evidenzbasierten Medizin
}

Eür die spezifische Immuntherapie (SIT) gab es bis vor kurzem wenig klinische Evidenz. Zwar belegten kontrollierte Studien ihre Wirksamkeit, diese genügten modernen Maßstäben jedoch nicht. Entsprechend unübersichtlich war die Marktlage mit über mehr als 6.500 Einzelpräparaten, ungeprüften Mischungen und unterschiedlichsten Dosierungen. Erst mit den Richtlinien der European Medicines Agency (EMA) zur klinischen Entwicklung von Therapieallergenen sei die SIT in der evidenzbasierten Medizin angekommen, erklärte Prof. Dr. Ulrich Wahn, Berlin: „Die Immuntherapie muss heute die gleiche Messlatte nehmen wie die Pharmakotherapie." Voraussetzung für die Zulassung sind demnach randomisierte plazebokontrollierte doppelblinde Multizenterstudien..

Eine Marktbereinigung erwartet Prof. Dr. Stefan Vieths vom Paul-Ehrlich-Institut in Langen von der Therapieallergene-Verordnung: Danach dürfen alle Präparate, für die bis Ende 2010 keine Zulassung beantragt wurde, nicht mehr verkauft werden. Für 123 Präparate liegt ein entsprechender Antrag vor.

Die 5-Gräser-Tablette Oralair ${ }^{\circledR}$ erfüllt bereits heute die Anforderungen der Therapieallergene-Verordnung. Die Sublingualtablette ist zugelassen zur Hyposensibilisierung bei gräserpollenbedingter allergischer Rhinitis. In umfangreichen Studienprogrammen geprüft, zeigte das Präparat eine Symptomreduktion um 39\% bei Kindern beziehungsweise 37\% bei Erwachsenen im ersten Jahr der Behandlung. Im dritten Jahr war der Symptomscore um $50 \%$ reduziert. Als optimale Dosis erwiesen sich 300 IR, berichtete Prof. Dr. Randolf Brehler aus Münster. Empfohlen wird ein Therapiebeginn vier Monate präsaisonal mit cosaisonaler Fortsetzung.

Michael Koczorek

Expert-Meeting „Die Dimension der evidenzbasierten SIT“, Berlin, 28. Januar 2011. Veranstalter: Stallergenes, Kamp-Lintfort
„N"-Kennzeichnung ausgesetzt Im Zuge des am 1. Januar 2011 in Kraft getretenen Arzneimittelmarktneuordnungsgesetzes (AMNOG) wird es auch Änderungen bei der Packungsgrößenverordnung geben. Der Gesetzgeber beabsichtigt langfristig, einheitliche Packungsgrößen einzuführen, die sich an der Therapiedauer orientieren. Für die spezifische Immuntherapie mit Tabletten wurde bislang noch keine endgültige Packungsgröße festgelegt. Die „N“-Kennzeichnung der Packungsgrößen wird daher für Oralair ${ }^{\circledR}$ vorübergehend ausgesetzt. Erst nach der Festlegung der endgültigen Packungsgrößen für die Tabletten-Immuntherapie wird Oralair $^{\circledR}$ wieder neu eingruppiert. Bis dahin kann es vorkommen, dass die „N“-Kennzeichnung auf den Oralair $^{\circledR}$-Packungen mit der Kennzeichnung in Arzneimitteldatenbanken nicht übereinstimmt. Dies beeinflusst jedoch nicht die Erstattungsfähigkeit. Die 5-Gräser-Tablette kann also wie gewohnt verordnet und an den Patienten abgegeben werden. red

Nach Informationen von Stallergenes, Kamp-Lintfort 